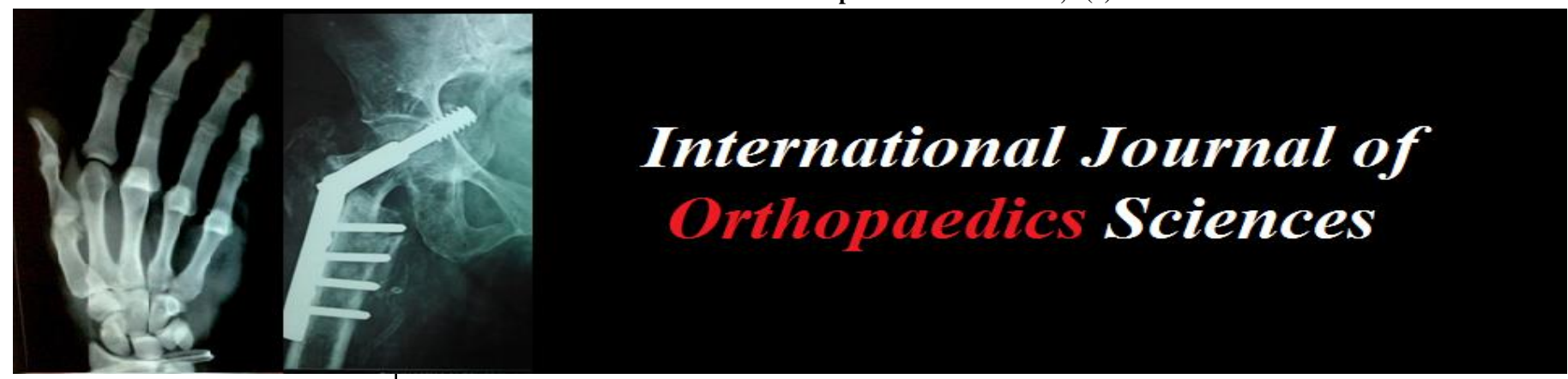

ISSN: $2395-1958$

IJOS 2019; 5(2): 230-234

(C) 2019 IJOS

www.orthopaper.com

Received: 09-02-2019

Accepted: 11-03-2019

Dr. Sathish Kumar Dake Assistant Professor, NRI Institute of Medical Sciences, Sangivalasa, Visakhapatnam, Andhra Pradesh, India

Dr. Ravi Kiran Kakumanu Assistant Professor, NRI Institute of Medical Sciences, Sangivalasa, Visakhapatnam, Andhra Pradesh, India

Dr. Mrudula Bheesetti M.B.B.S, NRI Institute of Medical Sciences, Sangivalasa, Visakhapatnam,

Andhra Pradesh, India
Correspondence

Dr. Ravi Kiran Kakumanu Assistant Professor, NRI Institute of Medical Sciences, Sangivalasa, Visakhapatnam, Andhra Pradesh, India

\section{Functional outcome of intracapsular fracture of neck of femur-osteosynthesis by cannulated cancellous screw fixation in adults}

\author{
Dr. Sathish Kumar Dake, Dr. Ravi Kiran Kakumanu and Dr. Mrudula \\ Bheesetti
}

DOI: https://doi.org/10.22271/ortho.2019.v5.i2e.26

\begin{abstract}
Introduction: Fractures of neck of femur have always presented great challenges to the orthopaedic surgeons. This remains, even today, an unsolved fracture as far as treatment and results are concerned. Results depend upon the extent of injury, timing of surgery and adequacy of reduction and fixation. Fixation with cannulated cancellous screws is usually adequate for femoral neck fractures. Lateral cortex plays a very important role in screw fixation.

Aims and objectives: To study the effectiveness of cannulated cancellous screw fixation for treatment of fracture of neck of femur in adults.

Materials and methods: This study was conducted at NRI Institute of Medical Sciences, Visakhapatnam, AP from March 2016 to February 2019. The patients with intracapsular fracture of neck of femur are evaluated with pre-operative X-rays of pelvis with both hips and X-ray of the concerned hip joint both in anteroposterior and lateral views and their outcome was evaluated postoperatively after fixation with cancellous screws. The outcome is evaluated in terms of pain relief, extent of ambulation achieved after surgery. The classifications we followed are Pauwel's and Garden's classification of fracture of neck of femur. The patients were followed up to one year to assess the functional outcome.

Observation and results: A good result was obtained in $66.1 \%$ of the patients, excellent in $23.2 \%$, fair in $3.8 \%$ and poor result in $6.9 \%$ of the patients. Complications such as Non-union \& avascular necrosis in one case, Non-union and Extrusion of screws in one case, Cut through of screws into articular surface leading to painful joint in one case. Most of the cases of intracapsular neck of femur were in the age group of 31-40 years. There was male preponderance as shown in this study (69\%).

Conclusion: By the usage of multiple cannulated cancellous lag screws, compression effect at the fracture site is achieved, it also avoids re-displacement and rotations. The implant occupies less volume in the small-sized femoral necks of South Indian patients allowing better osteosynthesis of intracapsular fracture of neck of femur. Multiple cannulated cancellous screw fixation for intracapsular fracture of neck of femur is an easy, safe \& useful procedure with encouraging results.
\end{abstract}

Keywords: Intracapsular fracture, osteosynthesis, internal fixation, cortico-cancellous screw

\section{Introduction}

Femoral neck fractures often are associated with multiple injuries and high rates of avascular necrosis and non-union. In 1931, Smith-Petersen using a tri-flanged nail, reported a series of open nailing in which he advocated reduction, impaction and internal fixation ${ }^{[1]}$. In 1989, Lars Rehnberg, Claes Olerud, from the University Hospital, Uppsala, Sweden recommended subchondral cannulated screw fixation for femoral neck fractures ${ }^{[2]}$. In 1997, V. K. Gautam, and colleagues of Department of Orthopaedics, Maulana Azad Medical College, New Delhi, India recommended management of displaced femoral neck fractures in young adults (15-50 years), primary open reduction and internal fixation of femoral neck fractures with three cancellous screws ${ }^{[3]}$. In 2010, Lin SQ et al. observed cannulated screw fixation and percutaneous autogenous bone marrow grafting is a more efficient method for accelerating healing of femoral neck fractures and reducing femoral head necrosis [3]. Even with undisplaced fracture of neck of femur, there is no assurance that a fracture will attain an excellent result ${ }^{[3]}$. 
Early anatomical reduction compression of the fracture and rigid internal fixation are used to promote union. An attempt has been made by this study to evaluate the role of multiple cancellous lag screws in internal fixation of intracapsular fracture of neck of femur. The aim of present study is to evaluate the effectiveness of cannulated cancellous screw fixation for treatment of fracture of neck of femur in adults. To study the rate of union (radiological and clinical) and the incidence of complications and compare the results of our study with the works reported.

2. Material and methods: The present study is carried out in the Department of Orthopaedics, NRI Institute of Medical Sciences, Visakhapatnam, AP during March 2016 to February 2019. All the patients were preoperatively assessed to grade the type of fracture by "Garden's Classification" and prepared for surgery. All fractures were reduced by Leadbetter (in flexion) method. A total of 28 cases of intracapsular fracture neck of femur in adults were treated after accurate reduction and rigid internal fixation under $\mathrm{X}$ - ray control with 3 partially threaded 6-5 mm Cannulated Cancellous screws.

\subsection{Inclusion Criteria}

1. Age 21-60 yrs.

2. Intracapsular neck of femur fractures only.

3. Independently mobile patients without neuromuscular disorders.

\subsection{Exclusion Criteria}

1. Age $<20$ yrs.

2. Extracapsular neck of femur fractures excluded.

3. Patient with polytrauma.

2.3. Surgical Technique: Internal fixation of intracapsular fracture of neck of femur by multiple cannulated cancellous screws.
2.4 Anaesthesia: Done under spinal anaesthesia, only a few cases were done under general anaesthesia.

2.5 Reduction: The patient was kept over the fracture table and fracture reduced by Leadbetter technique. The reduction was confirmed by both anteroposterior and lateral view of the hip.

2.6 Technique: After the reduction vertical incision was given over the lateral surface of the greater trochanter and extended distally up to $6-8 \mathrm{~cm}$, dissection was carried down through the skin and subcutaneous tissue and fascia lata was split.

Femoral cortex was approached by detaching vastus lateralis and reflecting it. Then, the lateral cortex was predrilled with 2 $\mathrm{mm}$ drill bit. Guide pins placed across the fracture from the lateral aspect of the femoral shaft parallel to the neck usually at a $135^{\circ}$ angle. One guide pin placed adjacent to the medial cortex at $135^{\circ}$ angle. Three guide pins placed at the middle of the head, one inferocentral, one anterior and one posterior, and driven within $5 \mathrm{~mm}$ of subchondral bone. Checked under $\mathrm{C}$-arm, both anteroposterior and lateral views; the guide pins measured to determine the correct screw length. After satisfactory position of the guide wires in the neck, drilling and tapping done over the guide wires with cannulated drill and cannulated tap respectively. Cannulated cancellous lag screws inserted over the guide wires by using the cannulated screw driver. Confirmation of adequate fixation done by checking under $\mathrm{C}$-arm both anteroposterior and lateral view. The screws should be within $5 \mathrm{~mm}$ of subchondral bone. If necessary washers were used to prevent the screw head shrinking and get the uniform compression at the fracture site. Haemostasis was secured. Wound closed in layers over the suction drain.

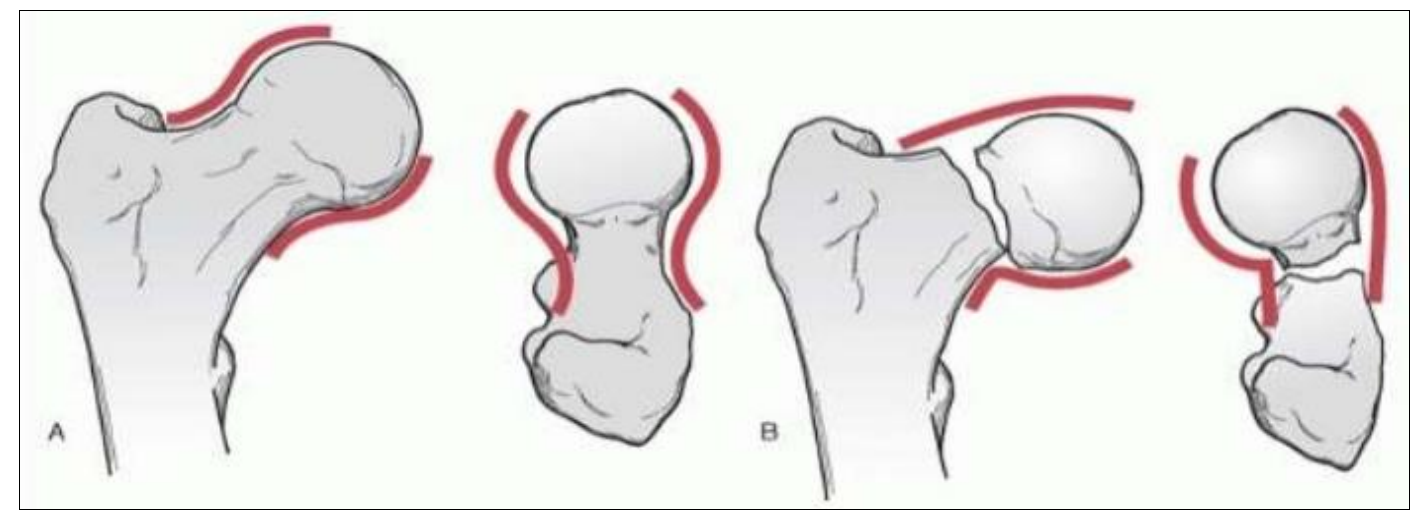

Fig 1: Evaluation of reduction

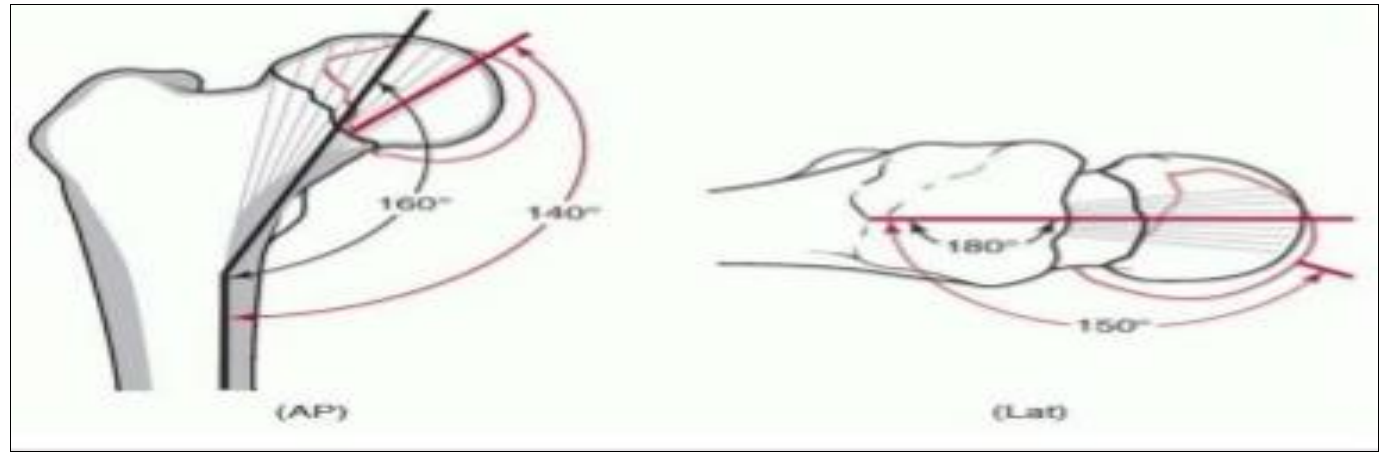

Fig 2: Garden alignment index 


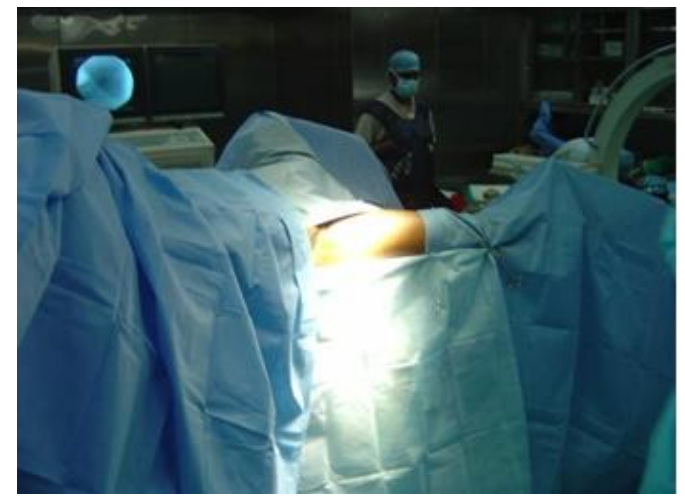

Fig 3: Patient positioning

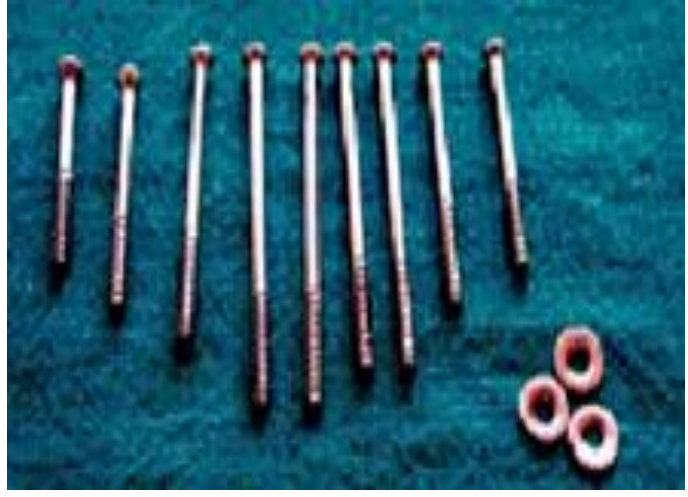

Fig 4: Cortico-cancellous screws with washers

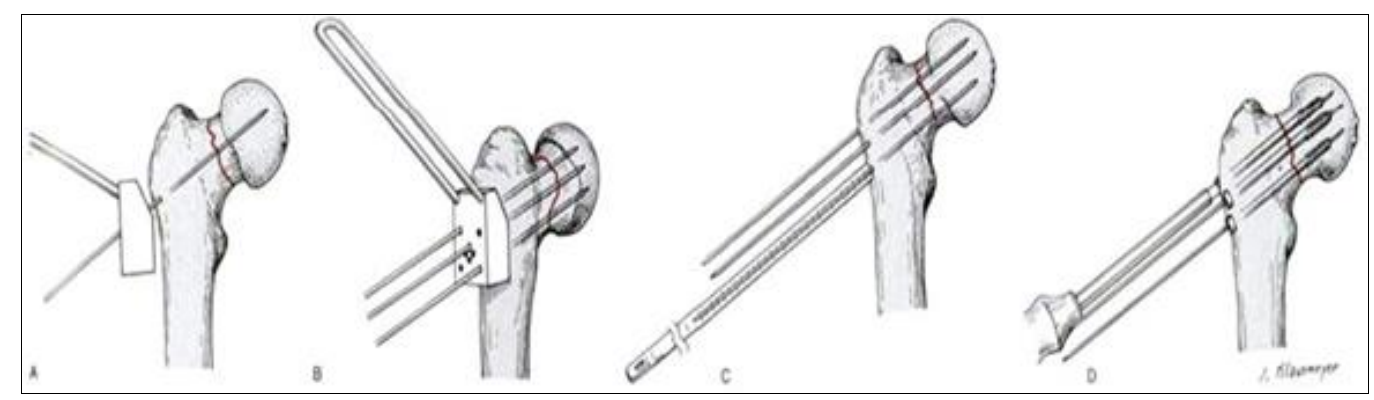

Fig 5: Internal fixation of a femoral neck fracture with a cannulated screw system: A, B, Reduction is confirmed, three parallel guide wires are placed using the guide and fluoroscopic control. C, Length of the wires is measured. D, Screws are inserted over the guide wires to the preselected depth

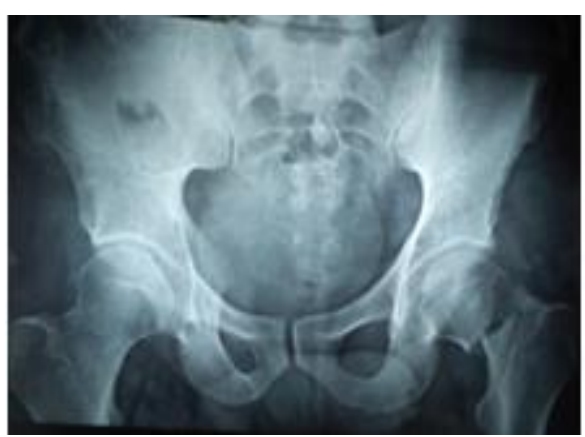

Fig 6: Pre-Operative Xray

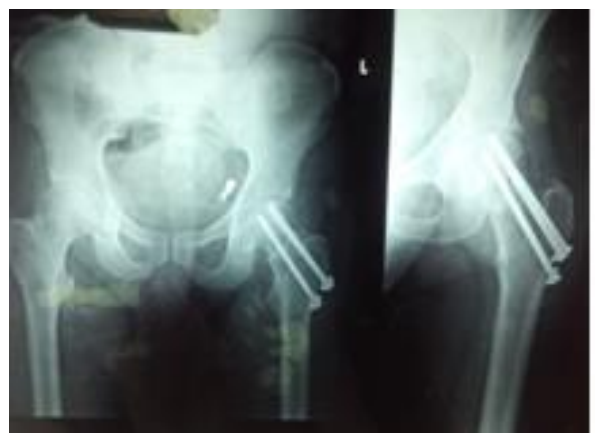

Fig 9: 6-Months Follow-Up

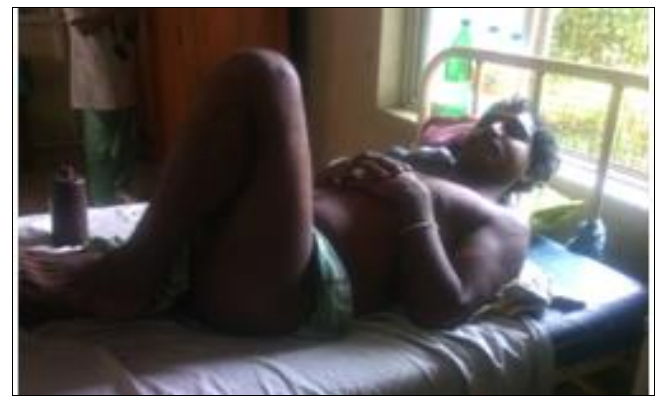

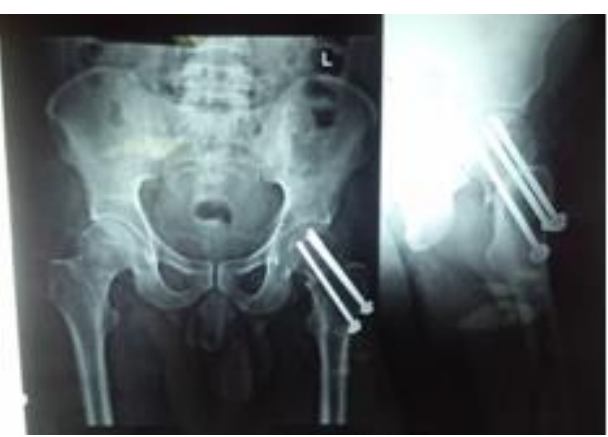

Fig 7: Immediate Post-Op Xray

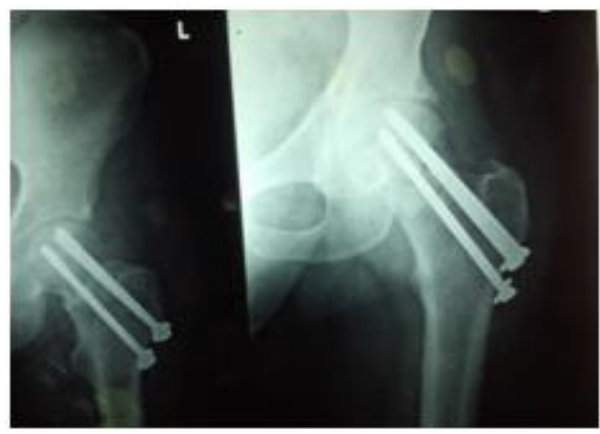

Fig 10: 1-Year Follow-Up

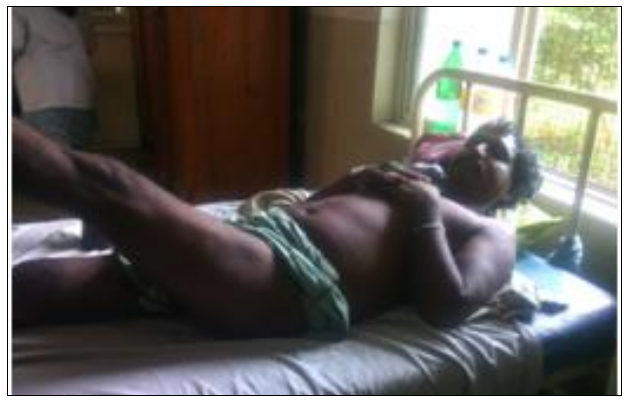

Fig 11: Post-operative range of motion $\sim 232 \sim$ 
3. Observation and results: In our study, there are 20 males and 8 females, Grading of results as per a six-point functional outcome scoring system for Asians after hip surgery ${ }^{[5]}$.

Table 1: Grading of results as per a six point functional outcome scoring system for Asians after hip surgery ${ }^{[4]}$

\begin{tabular}{|c|c|c|c|}
\hline Function & Score 0 & Score 0.5 & Score 1 \\
\hline Pain at hip & Moderate/severe & Mild & No pain \\
\hline Walking & Significant limp with aid & Mild limp with aid & No aid/No limp \\
\hline Independent mobility & Not possible & - & Possible \\
\hline Sitting cross legged & Not possible & Incomplete & Complete \\
\hline Squatting & Not possible & Incomplete & Complete \\
\hline Climbing stairs & Not possible & Using hand rails/bar support & Without any support \\
\hline
\end{tabular}

\section{Scoring Analysis}

Excellent: Score 5 or more.

Good: Score 4-4.5.

Fair: Score 2-3.5.

Poor: Score $<2$.

Table 2: Result of cases treated at NRI institute of medical sciences, Visakhapatnam

\begin{tabular}{|c|c|c|}
\hline Result & No. of cases & Percentage \\
\hline Excellent & 07 & $23.2 \%$ \\
\hline Good & 18 & $66.1 \%$ \\
\hline Fair & 01 & $3.8 \%$ \\
\hline Poor & 02 & $6.9 \%$ \\
\hline
\end{tabular}

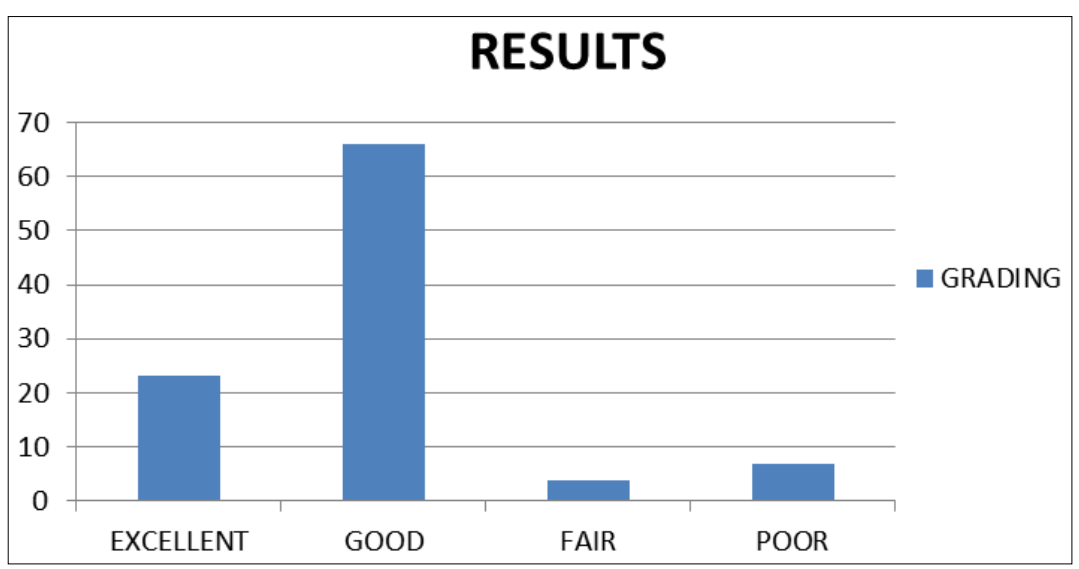

Graph 1: $66.1 \%$ of operated cases in our study achieved good result

\section{Complications}

1. One case of screw penetrating through the articular surface.

2. One case of nonunion with AVN.

3. One case of nonunion with loosening of screw

4. One case had absorption of the neck for which Girdle Stone excision arthroplasty done.

\section{Discussion}

Our important objective in the treatment of intracapsular fracture of the femur is to obtain stable osseous support of the femoral head on the femoral neck. The purpose of the fixation screws are to lock the fracture in a position in which the femoral neck gives bone-on-bone support to the femoral headneck fragment, to prevent posterior and varus migration of the femoral head, and to be parallel so as to maintain bone-onbone support as the fracture settles in the healing period.

\subsection{There are several reasons for use of a Cannulated screw system}

1. The smaller-diameter guide pins can be used to determine the screw position and length accurately.

2. Cannulated screw systems improve the accuracy of screw placement by supplying jigs that can place guide pins very accurately; and with parallel screws, excellent compression can be produced a traumatically by the lag effect of the screw

The total number of cases of Intracapsular fracture neck of femur followed are ${ }^{[28]}$. The cases were treated by multiple cannulated cancellous screws and followup from 6 months to 2 years. Percentage of male patients was higher than female patients. The commonest age group of the followed cases is between 31-40 years. The commonest radiological type of fracture is Garden's type IV followed by Type-II. In our study, Garden's type IV showed poor results when compared to others ${ }^{[6]}$. All the patients were explained regarding the precautions to be followed after surgery. One case developed absorption of the neck and loosening of the screws and for that Girdle Stone excision arthroplasty was done. One case developed collapse at the fracture site and extrusion of screws due to early weight-bearing and the case did not turn up for follow-up.

This series contains patients who are hard working labourers and sedentary females. The mechanism of injury in most cases is in the form of fall from height. There is also slightly violent injury leading to intracapsular fracture. The commonest radiological type of fracture is Garden's type IV followed by Type-II. In our study Gardens type IV showed poor results when compared to others ${ }^{[29]}$. All the patients 
were explained the precaution to be followed after surgery. The reduction of fracture was done by Lead better method without fail. The reduction was confirmed by an X-ray both Anteroposterior and lateral views. Through lateral approach, the fracture was fixed by multiple cannulated cancellous screws. In most of the cases the fixation of fracture was done by 2 or more than two to prevent rotation of the proximal fragment. The threaded portions of the screws were seen to cross the fracture line to get a better lag effect. A similar study was conducted V. K. Gautam, S. Anand and B. K. Dhaon of department of Orthopaedics, Maulana Azad Medical College, New Delhi, India, V. K. Gautam ${ }^{[8]} \&$ associates conducted a study on management of displaced femoral neck fractures in young adults between age group 15 - 50 years. Open reduction of the fracture site and fixation with three cancellous screws was done. Union of fracture was seen in $96 \%$ of patients, non-union reported in only $4 \%$ of cases, and evidence of avascular necrosis was seen in three cases (12 per cent). Lars Rehnberg, Claes Olerud ${ }^{[9]}$ From the University Hospital, Uppsala, Sweden conducted a study on subchondral screw fixation for femoral neck fractures, in their study all fractures healed within 12 months, and there were no cases of early loosening or non-union. The result of both these studies is similar with our study.

Table 3: Comparison of results

\begin{tabular}{|c|c|c|c|c|}
\hline & Excellent & Good & Fair & Poor \\
\hline Bhava RJ Satish et al. & $78.2 \%$ & $20.3 \%$ & - & $1.5 \%$ \\
\hline Present study & $23.2 \%$ & $66.1 \%$ & $3.8 \%$ & $6.9 \%$ \\
\hline
\end{tabular}

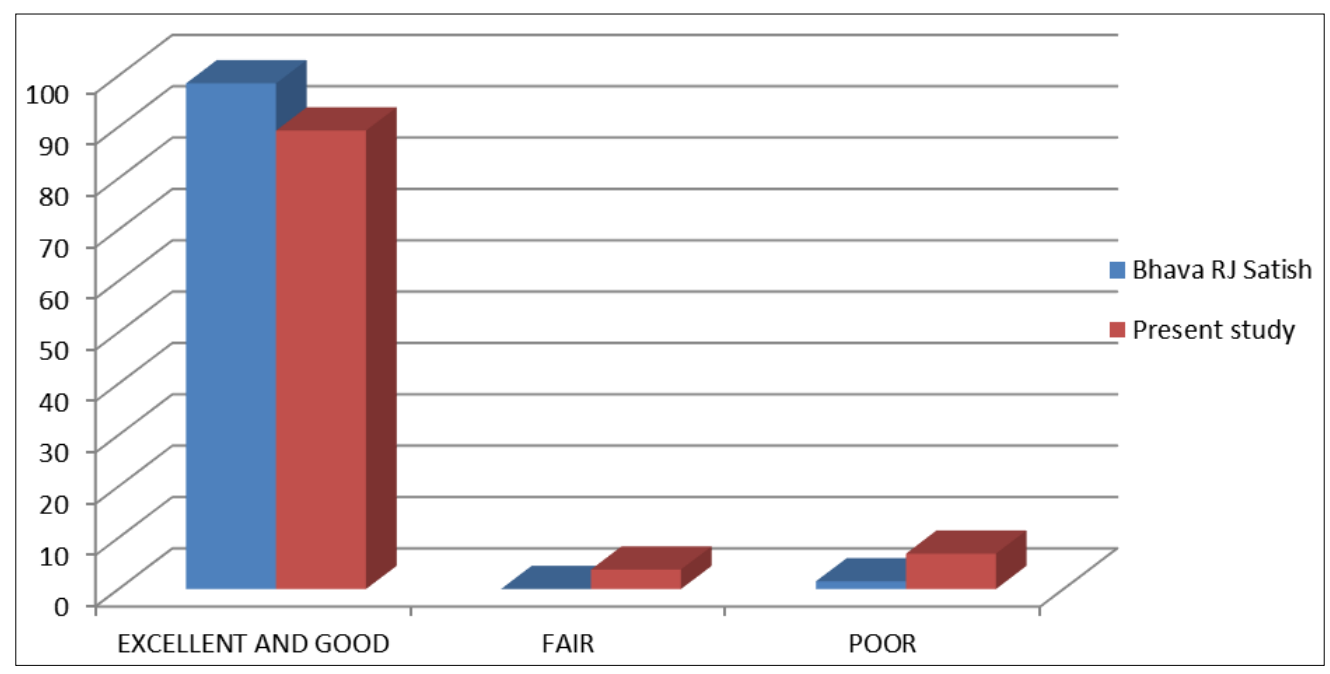

Graph 2: Comparison of results

\section{Conclusion}

The most of the cases of intracapsular fracture neck of femur were in the age group of 31- 40 years. There was male preponderance as shown in this study of intracapsular fracture neck of femur. In our study the side of hip which was frequently fractured is right hip, of 28 patients, 18 patients fractured right hip. The nature of violence in this study shows, mainly, fall from a height. This injury usually not associated with any other injuries. In our institute accurate reduction and rigid internal fixation of intracapsular fracture neck of femur was done with the help of C-Arm and the results were encouraging even up to the age of 60 years. By early mobilization of the patients the complications of prolonged immobilisation like thromboembolism, hypostatic pneumonia were avoided. By the usage of multiple cannulated cancellous lag screws, compression effect at the fracture site is achieved, it also avoids redisplacement and rotations. The implant occupies less volume in the small sized femoral necks of South Indian Patients allowing better osteosynthesis of intracapsular fracture neck of femur.

Thus Multiple cannulated cancellous screw fixation for intracapsular fracture neck of femur is an easy, safe \& useful procedure with encouraging results.

\section{References}

1. Richard KF. Factures and dislocations. Chapter 23. Ramon GB, Richard KF, David T, eds. Mosby 1993; 2:783-854.
2. Rehnberg L, Olerud C, Hellquist E. Internal fixation of femoral neck fractures. JBJS (Br). 1991; 73-B:16-19.

3. Gautam VK, Anand S, Dhaon BK. Management of displaced femoral neck fractures in young adults (a group at risk). Injury. 1998; 29(3):215-218.

4. Lin SQ, Peng LP, Yao ZC. Case-control study on cannulated screw fixation and percutaneous autogenous bone marrow grafting for the treatment of femoral neck fractures. Zhongguo Gu Shang. 2010; 23(9):675-678.

5. Satish BRJ, Atmakuri VR, Ramalingam K et al. Four quadrant parallel peripheral screw fixation for displaced femoral neck fractures in elderly patients. Indian Journal of Orthopaedics. 2013; 47(2):174-181.

6. Skála-Rosenbaum J, Dzupa V, Bartonícek J et al. Osteosynthesis of intracapsular femoral neck fractures. Rozhl Chir. 2005; 84(6):291-298. 